\title{
Prenatal smoking intervention in managed care settings: the Kaiser Permanente Southern California prenatal smoking project
}

\author{
Virginia P Quinn
}

Smoking during pregnancy is the leading preventable cause of perinatal morbidity and mortality. ${ }^{1}$ The dissemination of health warnings has been associated with a decline in prenatal smoking over the past several decades. $^{23}$ Yet, approximately one fourth of US women of childbearing age currently smoke and only a third of them will quit for pregnancy. ${ }^{3}$ Women smoking at the time they become pregnant are known as pre-pregnancy smokers. Most women who stop smoking for pregnancy do so on their own before they initiate prenatal care and are called spontaneous quitters. Compared to women still smoking (intake smokers), spontaneous quitters more often are younger, having their first baby, have completed more years of education, are more confident about quitting, and are less addicted, as measured by the number of cigarettes smoked per day before pregnancy. Consequently, as the proportion of spontaneous quitters grows, characteristics of women still smoking present an increasingly difficult challenge to cessation intervention.

Although absolute quit rates have rarely exceeded $25 \%$, previous research found prenatal smoking intervention increases cessation by $70 \%$ and is a cost effective prevention strategy. ${ }^{5}$ The first generation of intervention improved cessation rates through the systematic identification of smokers and by tailoring culturally relevant program content to pregnancy. The Kaiser Permanente prenatal smoking trial attempted to enhance the effectiveness of earlier programs through matching interventions to smokers' stage of readiness to change and barriers to cessation, particularly level of addiction. ${ }^{7}$ The study compared three interventions of varying intensity that were delivered in addition to usual care advice. A third of the subjects received only a self help booklet, "Living smoke-free", a third received the booklet and had access by phone to a computerised, interactive voice response cessation program, and a third received the booklet and up to six counselling calls from nurse educators trained in motivational interviewing. At the end of pregnancy, about $20 \%$ of women in all groups were biochemically confirmed as abstinent. Furthermore, no differential intervention effects were found by stage of change, level of addiction, or degree of self efficacy. In contrast to the modest intervention related cessation rate, we found a spontaneous quit rate of $60.4 \%$ in this population of prepregnancy smokers.
Consistent with national recommendations, ${ }^{9}$ results support the provision of a low cost, self help cessation program booklet in addition to usual care advice from prenatal providers. "Living smoke-free" is written at an eighth grade (age 13 to 14 years) reading level, yet presents materials in a sophisticated format. Messages tailored to pregnancy are delivered by four racially/ethnically diverse characters, each representing a stage of readiness to change. A process evaluation revealed high use and satisfaction with the booklet in a socioeconomically diverse population of prenatal smokers. We believe it is important to deliver the program through a special introduction in order to distinguish it from other materials received at the start of prenatal care. We chose to mail the program separately under a cover letter from smokers' prenatal providers. While more effective program intervention remains to be developed, at this time managed care organisations can help address a serious threat to maternal, infant, and child health through systematic identification of prenatal smokers and the widespread dissemination of an inexpensive and easily implemented prenatal smoking cessation program intervention.

The Kaiser Permanente prenatal smoking cessation trial was supported by a grant from the Robert Wood Johnson
Foundation, Smoke-Free Families Initiative awarded to Daniel Ershoff. Many thanks are due to Kaiser Permanente Southern California staff for their assistance in conducting the research and implementing the prenatal smoking cessation program.

1 US Department of Health and Human Services. The health benefits of smoking cessation. A report of the Surgeon General, 1990. Rockville, Maryland: Public Health Service, Centers 1990. Rockville, Maryland: Public Health Service, Centers
for Disease Control, Office on Smoking and Health, 1990. for Disease Control, Office on Smoking and
(DHHS Publication No (CDC) 90-8416.)

2 Centers for Disease Control and Prevention. Best practices for comprehensive tobacco control programs-August 1999. Atlanta: US Department of Health and Human Services, Centers for Disease Control and Prevention, National Center for Chronic Disease Prevention and National Center for Chronic Disease Prevention and Health

3 Floyd RL, Rimer BK, Giovino GA, et al. A review of smoking in pregnancy: effects on pregnancy outcomes and cessation efforts. Ann Rev Public Health 1993;14:379-411.

4 Kendrick JS, Merritt RK. Women and smoking: an update for the 1990s. Am f Obstet Gynecol 1996;175:528-35.

5 Mullen PD. Maternal smoking during pregnancy and evidence-based intervention to promote cessation. Prim Care 1999;26:577-91.

6 Marks JS, Koplan JP, Hogue CJ, et al. A cost-benefit/costeffectiveness analysis of smoking cessation for pregnant women. Am f Prev Med 1990;6:282-9.

7 Ershoff DE, Quinn VP, Boyd NR, et al. The Kaiser Permanente prenatal smoking cessation trial: when more isn't better, what is enough? Am f Prev Med 1999;17:161-8.

8 Anon. Living-smoke free. San Bruno, CA:Krames Communications/StayWell Co, 1996.

9 The Smoking Cessation Clinical Practice Guideline Panel and Staff. The Agency for Health Care Policy and Research smoking cessation clinical practice guideline. fAMA 1996; 275:1270-80. 